\title{
CEP3 and CEP17 DNA probe potential in the genetic diagnosis and prognostic prediction of esophageal squamous cell cancer
}

\author{
MADINIYAT NIYAZ ${ }^{1}$, ABLAJAN ABDURAHMAN ${ }^{2}$, ABDUGHENI TURGHUN $^{2}$ and IDIRIS AWUT ${ }^{2}$ \\ ${ }^{1}$ Xinjiang Esophageal Cancer Research Institute, Medical Research Center; ${ }^{2}$ Department of Thoracic Surgery, \\ First Affiliated Hospital of Xinjiang Medical University, Urumqi, Xinjiang 830054, P.R. China
}

Received November 10, 2014; Accepted November 25, 2015

DOI: $10.3892 / \mathrm{etm} .2016 .3080$

\begin{abstract}
The aim of the present study was to investigate the clinical application of molecular pathological diagnosis for the prognosis of Kazakh patients with esophageal squamous cell carcinoma (ESCC) using centromere enumeration probes (CEPs) for chromosomes 3 and 17. A total of 40 patients with ESCC that had received radical surgical treatment and 10 healthy control participants were enrolled in the present study. Touch preparations of fresh cancerous and normal tissues were completed and fluorescence in situ hybridization (FISH) was performed to count the copy numbers of CEP 3 and 17, and abnormalities were analyzed, in comparison with routine pathological diagnoses. FISH analysis demonstrated that abnormal copy numbers of CEP 3 and 17 (aneuploidy) were detected in all 40 patients with ESCC. CEP 3 and 17 polyploidy rates differed significantly between poorly differentiated, moderately differentiated and well-differentiated ESCC groups $(\mathrm{P}<0.05)$ : Well-differentiated, 35.38 and $30.92 \%$; moderately differentiated, 55.81 and $44.43 \%$; and poorly differentiated, 76.26 and $71.90 \%$, respectively. Furthermore, polyploidy rates were significantly increased in the group with lymph node metastasis, as compared with the group without (CEP 3, P=0.0001; CEP 17, P=0.012). Variations in the copy numbers of CEP 3 and 17 were demonstrated to be correlated with the level of differentiation and lymph node metastasis in patients with ESCC. Therefore, the present results indicate that DNA probes may be used to predict prognostic factors in patients with ESCC. Furthermore, FISH technology is an objective and qualitative method that is capable of detecting
\end{abstract}

Correspondence to: Professor Idiris Awut, Department of Thoracic Surgery, First Affiliated Hospital of Xinjiang Medical University, 137 Liyushan South Road, Urumqi, Xinjiang 830054, P.R. China

E-mail: edrs5@yahoo.com

Abbreviations: FISH, fluorescence in situ hybridization; EC, esophageal cancer; ESCC, esophageal squamous cell carcinoma; CEP, centromere of chromosome; SSC, standard saline citrate

Key words: DNA probe, fluorescence in situ hybridization, aneuploid, esophageal cancer, Kazakh patients variations in CEP 3 and 17; therefore, FISH may be used in the genetic diagnosis of ESCC in Kazakh patients.

\section{Introduction}

Esophageal cancer (EC) is among the most common types of cancer associated with the digestive system. Due to a lack of efficient early diagnostic measurements, the majority of patients with EC are in an advanced stage when treatment is initiated, which is associated with a poor prognosis. Mortality rates of patients with EC are high ( $>300,000$ patients per year), and the incidence of EC among Kazakh people in the Xinjiang Uyghur Autonomous Region of northwest China is $155.9 / 100,000$, which is higher than any other ethnic group in China (1-3).

Conventional methods of pathological diagnosis provide crucial information regarding tumor differentiation and the morphological characteristics $(4,5)$ in patients with EC. However, clinical diagnosis and decisions on treatment are difficult due to various limitations, including tissue disfigurement following extrusion, inadequate biopsy depth and discrepancies between pathological diagnosis and actual diagnosis. Therefore, a more objective and quantitative method is required to improve the accuracy of EC diagnosis (6).

Previous studies have suggested that the incidence and evolution of EC is associated with various chromosomal anomalies $(7,8)$. During carcinogenesis, cells undergo molecular cytogenetic changes prior to any alterations in morphology. Nuclear chromosome abnormality, which is observed in cancer cells, is an early event during the process of tumorigenesis, and it has become the objective index for determining cancerous cells $(9,10)$. Nuclear aneuploidy is a prevalent feature of various types of cancer, including EC $(9,10)$; therefore, the detection of aneuploidy, which is usually found in aneusomic nuclei, may be used to detect cancerous cells. Fluorescence in situ hybridization (FISH) technology is a rapid and sensitive method used for the detection of aneusomy on a specific chromosome (11). FISH has been utilized for the diagnosis of various types of cancer with high sensitivity and specificity, including hematological malignancies and lung, breast and kidney cancer $(12,13)$. The advantage of FISH is that it is an objective and quantitative method for identifying cancerous cells. Previous studies have 
demonstrated that the priority of FISH in cancer detection is higher than conventional cytology and, therefore, may be advantageous to the early diagnosis of various types of cancer $(14,15)$.

In the present study, chromosomal loci centromere of chromosome (CEP) 3 and 17 were selected to fabricate the DNA probe as they have an increased aberration rate in esophageal and lung cancer $(16,17)$. In order to analyze the clinical application of FISH and any correlations between the prognosis of patients in the diagnosis of ESCC, 40 Kazakh patients with esophageal squamous cell carcinoma (ESCC) underwent FISH examination and conventional pathological diagnosis using surgical samples.

\section{Patients and methods}

Patients. Between June 2011 and September 2012, 40 Kazakh patients with ESCC were admitted to the Department of Thoracic Surgery at The First Affiliated Hospital of Xinjiang Medical University (Urumqi, China) and underwent surgical resection. Among the 40 patients enrolled in the present study, 34 were male and 6 were female, with an average age of 57.4 years (Table I). None of the patients had previously received any preoperative radiotherapy, chemotherapy or other treatment. Pathology was graded according to the 7th American Joint Committee on Cancer staging manual (18). Final post-operative pathological diagnosis confirmed ESCC in all 40 cases, including well differentiated $(n=13)$, moderately differentiated $(n=16)$ and poorly differentiated tumors $(\mathrm{n}=11)$. Lymph node metastasis was detected in 55\% (22/40) of cases; therefore, $45 \%$ (18/40) of the cases enrolled in the present study were non-metastatic. A total of 10 esophageal tissue samples ( $>5 \mathrm{~cm}$ distant from the tumor) were harvested as normal controls from healthy individuals. Informed consent was obtained from all patients.

FISH method. Touch preparations of cells were performed on glass slides from fresh specimens and air-dried for $24 \mathrm{~h}$ at room temperature and were subsequently stored at $-80^{\circ} \mathrm{C}$ in preparation for FISH. The same specimens were stained with hematoxylin and eosin for pathological evaluation (Beijing Zhongshan Jinqiao Biotechnology Co., Ltd., Beijing, China).

Cells were denatured with $70 \%$ formaldehyde at $74^{\circ} \mathrm{C}$ in a water bath and subsequently washed twice with standard saline citrate (SSC; Abbott Molecular, Inc., Des Plaines, IL, USA) at room temperature. Slides were dehydrated through a graded ethanol series (70,85 and $100 \%)$ prior to the application of $10 \mu 1$ hybridization solution, containing $1 \mu 1$ orange fluorescently-labeled CEP 3 probe, $1 \mu 1$ green fluorescently-labeled CEP 17 probe, $7 \mu 1$ hybridization buffer and $1 \mu \mathrm{l}$ double-distilled water (all Abbott Molecular, Inc.). Slides were covered with a cover slip and sealed with rubber cement (Fixogum; Marabu GmbH \& Co. KG, Tamm, Germany). Following incubation for $16 \mathrm{~h}$ at $42^{\circ} \mathrm{C}$ in a humidity-controlled chamber, the slides were washed with $\mathrm{SSC}$ at $74^{\circ} \mathrm{C}$ and at room temperature for $2 \mathrm{~min}$. Subsequently, $5 \mu 1$ diamidinophenylindole (DAPI II; Abbott Molecular, Inc.) was applied to each spot and covered with a cover slip.

Two fluorescently-labeled probes were used, Cy3 and FITC (Thermo Fisher Scientific, Inc., Waltham, MA, USA),
Table I. Clinical characteristics of the patients with esophageal squamous cell carcinoma.

\begin{tabular}{|c|c|c|c|}
\hline Case & Age (years) & Gender & Pathology \\
\hline 1 & 71 & $\mathrm{M}$ & T1bNOM0, IA \\
\hline 2 & 75 & M & T2N0M0, IB \\
\hline 3 & 71 & M & T3N0M0, IIA \\
\hline 4 & 55 & $\mathrm{M}$ & T2N0M0, IB \\
\hline 5 & 58 & M & T3N1M0, IIIA \\
\hline 6 & 54 & $\mathrm{~F}$ & T3N0M0, IIB \\
\hline 7 & 69 & M & T2N0M0, IB \\
\hline 8 & 55 & $\mathrm{M}$ & T3N2M0, IIIB \\
\hline 9 & 62 & M & T2NOM0, IB \\
\hline 10 & 45 & F & T3N1M0, IIIA \\
\hline 11 & 54 & $\mathrm{M}$ & T2N0M0, IB \\
\hline 12 & 60 & M & T2NOM0, IIA \\
\hline 13 & 57 & $\mathrm{M}$ & T1N0M0, IA \\
\hline 14 & 70 & M & T3N2M0, IIIB \\
\hline 15 & 58 & $\mathrm{~F}$ & T2N0M0, IIA \\
\hline 16 & 56 & M & T3N1M0, IIIA \\
\hline 17 & 73 & M & T2N1M0, IIB \\
\hline 18 & 68 & M & T3N1M0, IIIA \\
\hline 19 & 40 & $\mathrm{M}$ & T1bN0M0, IA \\
\hline 20 & 43 & $\mathrm{~F}$ & T2N1M0, IIB \\
\hline 21 & 54 & $\mathrm{M}$ & T3N2M0, IIIB \\
\hline 22 & 47 & M & T3N1M0, IIIA \\
\hline 23 & 44 & M & T3N1M0, IIIA \\
\hline 24 & 56 & M & T2N1M0, IIB \\
\hline 25 & 42 & M & T3N1M0, IIIA \\
\hline 26 & 45 & $\mathrm{M}$ & T3N0M0, IIB \\
\hline 27 & 50 & M & T3N1M0, IIIA \\
\hline 28 & 56 & $\mathrm{~F}$ & T2N0M0, IB \\
\hline 29 & 72 & M & T3N1M0, IIIA \\
\hline 30 & 65 & M & T2N1M0, IIB \\
\hline 31 & 67 & $\mathrm{~F}$ & T3N1M0, IIIA \\
\hline 32 & 52 & $\mathrm{M}$ & T1aNOM0, IA \\
\hline 33 & 50 & M & T3N1M0, IIIA \\
\hline 34 & 48 & $\mathrm{M}$ & T3N1M0, IIIA \\
\hline 35 & 62 & $\mathrm{M}$ & T2N1M0, IIB \\
\hline 36 & 50 & $\mathrm{M}$ & T2N0M0, IB \\
\hline 37 & 63 & M & T3N0M0, IIB \\
\hline 38 & 55 & M & T1bN0M0, IA \\
\hline 39 & 72 & $\mathrm{M}$ & T3N1M0, IIIA \\
\hline 40 & 50 & M & T2N1M0, IIB \\
\hline
\end{tabular}

Pathology graded according to the 7th American Joint Committee on Cancer staging manual (18). M, male; F, female.

and each of the protocols included at least one normal esophageal tissue as a control. FISH signal analysis was performed according to the kit instructions (Vysis CEP 3 (D3Z1) SpectrumOrange Probe Kit and CEP 17 Probe Kit; Abbott Molecular, Inc.) A fluorescence microscope and an image acquisition and analysis system (DM6000; Leica 
Table II. Results of fluorescence in situ hybridization in normal esophageal tissue.

\begin{tabular}{|c|c|c|c|c|}
\hline \multirow[b]{2}{*}{ Case } & \multicolumn{3}{|c|}{ Aneuploidy (CEP 3/CEP 17) } & \multirow[b]{2}{*}{ Multiple copies } \\
\hline & 2 copies & 3 copies & 4 copies & \\
\hline 1 & $96 / 96$ & $1 / 2$ & $3 / 2$ & $4 / 4$ \\
\hline 2 & $97 / 95$ & $2 / 3$ & $1 / 2$ & $3 / 5$ \\
\hline 3 & $97 / 97$ & $2 / 2$ & $1 / 1$ & $3 / 3$ \\
\hline 4 & $98 / 98$ & $2 / 2$ & 0 & $2 / 2$ \\
\hline 5 & $96 / 97$ & $3 / 2$ & $1 / 1$ & $4 / 3$ \\
\hline 6 & $96 / 95$ & $4 / 2$ & $0 / 3$ & $4 / 5$ \\
\hline 7 & $95 / 95$ & $2 / 2$ & $3 / 3$ & $5 / 5$ \\
\hline 8 & $95 / 96$ & $3 / 2$ & $2 / 2$ & $5 / 4$ \\
\hline 9 & $93 / 94$ & $3 / 3$ & $4 / 3$ & $7 / 6$ \\
\hline 10 & $97 / 95$ & $2 / 3$ & $1 / 2$ & $3 / 5$ \\
\hline
\end{tabular}

CEP, centromere of chromosome.
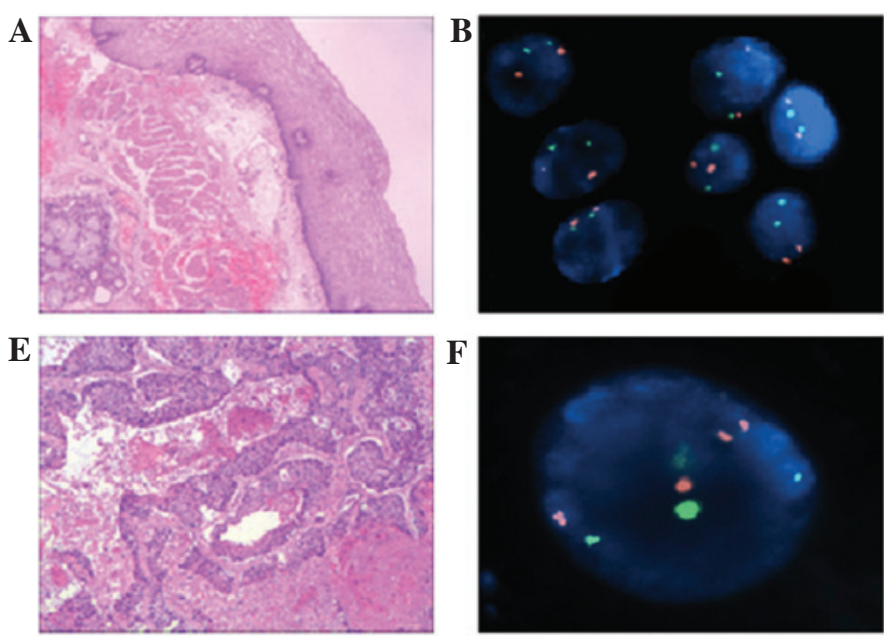
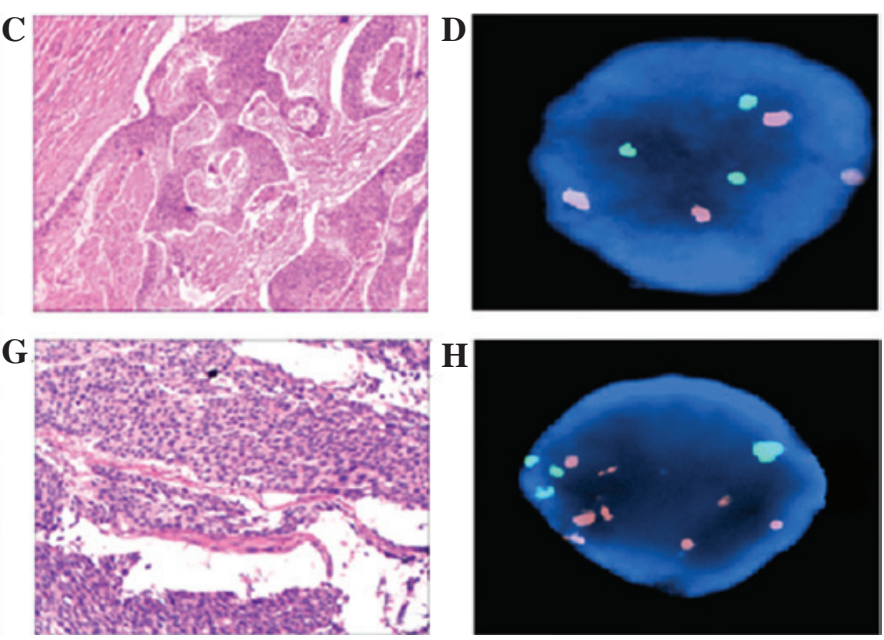

Figure 1. Comparison of hematoxylin and eosin (H\&E) staining and fluorescence in situ hybridization (FISH) in normal and abnormal esophageal tissues. (A) Normal esophageal membranes following H\&E staining (magnification, 40). (B) Normal representative nuclei carrying 2 copies of the centromeres of chromosomes (CEP) 3 (orange) and 17 (green). (C) Well-differentiated esophageal squamous cell carcinoma (ESCC) following H\&E staining (magnification, 40). (D) FISH representative nuclei carrying 3 copies of CEP 3 and 3 copies of CEP 17. (E) Moderately differentiated ESCC following H\&E staining (magnification, 40). (F) FISH representative nuclei carrying 4 copies of CEP 3 and 3 copies of CEP 17. (G) Poorly differentiated ESCC H\&E staining (magnification, 100). (H) FISH representative nuclei carrying 7 copies of CEP 3 and 4 copies of CEP 17.

Microsystems GmbH, Wetzlar, Germany) was used to determine the signal count. Results were analyzed by two individual statisticians. All cells were evaluated, with the exception of damaged cells or those with overlapping nuclei. A total of 100 nuclei were counted from each patient, and the total number of centromeric signals was recorded. Cells were deemed positive for aneuploidy when the percentage of hyperdisomic nuclei with $>3$ copies of at least one nucleus was $>10 \%$.

Statistical analysis. SPSS 17.0 statistical software (SPSS, Inc., Chicago, IL, USA) was used to perform statistical analyses in the present study. The Rank sum test and t-test were used to analyze the data from each group. Throughout the study, $\mathrm{P}<0.05$ was considered to indicate a statistically significant difference.

\section{Results}

Polyploidy rates of the centromeres of chromosomes 3 and 17 were detected using CEPs and FISH analysis. The percentage of diploid CEP3 and 17 was $>93 \%$ in the control group (Table II). Abnormal polyploidy copy numbers of CEP3 and 17 were detected in all 40 ESCC specimens (Fig. 1).

CEP3 and CEP17 aberrations correlated with lymph node metastasis. Average mutation rates of CEP 3 and 17 were $61.2 \%$ and $50.95 \%$, respectively (Table III). The sensitivity and specificity of the FISH results were $100 \%$ with no false positives or negatives, calculated according to the final pathological diagnosis. Lymph node metastasis was detected in 55\% of cases, therefore, $45 \%$ of the cases enrolled in the present study were non-metastatic. Furthermore, polyploidy rates were 
Table III. Results of fluorescence in situ hybridization in esophageal cancer tissue.

\begin{tabular}{|c|c|c|c|c|c|c|}
\hline \multirow[b]{2}{*}{ Case } & \multirow[b]{2}{*}{ Pathology } & \multicolumn{4}{|c|}{ Aneuploidy (CEP 3/CEP 17) } & \multirow[b]{2}{*}{ Multiple copies } \\
\hline & & 2 copies & 3 copies & 4 copies & $\geq 5$ copies & \\
\hline 1 & WDSCC & $45 / 39$ & $25 / 31$ & $20 / 22$ & $10 / 8$ & $55 / 61$ \\
\hline 2 & WDSCC & $71 / 73$ & $19 / 15$ & $10 / 12$ & $0 / 0$ & $29 / 27$ \\
\hline 3 & WDSCC & $73 / 77$ & $17 / 12$ & $9 / 11$ & $1 / 0$ & $27 / 23$ \\
\hline 4 & WDSCC & $65 / 63$ & $21 / 16$ & $11 / 12$ & $3 / 9$ & $35 / 37$ \\
\hline 5 & WDSCC & $60 / 72$ & $28 / 12$ & $5 / 8$ & $7 / 8$ & $40 / 28$ \\
\hline 6 & WDSCC & $82 / 80$ & $17 / 15$ & $0 / 5$ & $1 / 0$ & $18 / 20$ \\
\hline 7 & WDSCC & $76 / 74$ & $13 / 11$ & $6 / 7$ & $5 / 8$ & $24 / 26$ \\
\hline 8 & WDSCC & $72 / 68$ & $13 / 15$ & $7 / 9$ & $8 / 8$ & $28 / 32$ \\
\hline 9 & WDSCC & $66 / 72$ & $20 / 17$ & $10 / 9$ & $4 / 2$ & $34 / 28$ \\
\hline 10 & WDSCC & $59 / 68$ & $26 / 21$ & $6 / 7$ & $9 / 4$ & $41 / 32$ \\
\hline 11 & WDSCC & $60 / 65$ & $21 / 19$ & $11 / 8$ & $8 / 8$ & $40 / 35$ \\
\hline 12 & WDSCC & $50 / 57$ & $20 / 17$ & $25 / 20$ & $5 / 6$ & $50 / 43$ \\
\hline 13 & WDSCC & $71 / 74$ & $11 / 11$ & $10 / 10$ & $8 / 5$ & $29 / 26$ \\
\hline 14 & MDSCC & $41 / 59$ & $11 / 12$ & $36 / 20$ & $12 / 9$ & $59 / 41$ \\
\hline 15 & MDSCC & $38 / 56$ & $25 / 17$ & $28 / 20$ & $9 / 7$ & $62 / 44$ \\
\hline 16 & MDSCC & $43 / 59$ & $28 / 18$ & $13 / 12$ & $16 / 11$ & $57 / 41$ \\
\hline 17 & MDSCC & $64 / 65$ & $18 / 13$ & $12 / 15$ & $6 / 7$ & $36 / 35$ \\
\hline 18 & MDSCC & $38 / 57$ & $26 / 29$ & $28 / 11$ & $8 / 3$ & $62 / 43$ \\
\hline 19 & MDSCC & $31 / 56$ & $36 / 20$ & $19 / 12$ & $16 / 12$ & $69 / 44$ \\
\hline 20 & MDSCC & $52 / 78$ & $32 / 17$ & $13 / 4$ & $3 / 1$ & $48 / 22$ \\
\hline 21 & MDSCC & $60 / 70$ & $9 / 11$ & $12 / 12$ & $9 / 7$ & $40 / 30$ \\
\hline 22 & MDSCC & $45 / 55$ & $26 / 21$ & $12 / 9$ & $17 / 15$ & $55 / 45$ \\
\hline 23 & MDSCC & $39 / 46$ & $45 / 39$ & $9 / 13$ & $7 / 2$ & $61 / 54$ \\
\hline 24 & MDSCC & $48 / 57$ & $25 / 20$ & $19 / 16$ & $8 / 7$ & $52 / 43$ \\
\hline 25 & MDSCC & $44 / 54$ & $31 / 26$ & $23 / 15$ & $3 / 5$ & $56 / 46$ \\
\hline 26 & MDSCC & $45 / 52$ & $25 / 25$ & $12 / 8$ & $18 / 15$ & $55 / 48$ \\
\hline 27 & MDSCC & $24 / 24$ & $55 / 36$ & $9 / 30$ & $12 / 10$ & $76 / 76$ \\
\hline 28 & MDSCC & $15 / 26$ & $35 / 44$ & $20 / 20$ & $30 / 10$ & $85 / 74$ \\
\hline 29 & MDSCC & $80 / 75$ & $19 / 15$ & $1 / 10$ & $0 / 0$ & $20 / 25$ \\
\hline 30 & PDSCC & $30 / 38$ & $27 / 21$ & $24 / 26$ & $19 / 15$ & $70 / 62$ \\
\hline 31 & PDSCC & $25 / 28$ & $40 / 40$ & $25 / 23$ & $10 / 9$ & $75 / 72$ \\
\hline 32 & PDSCC & $21 / 27$ & $30 / 23$ & $39 / 40$ & $10 / 10$ & $79 / 73$ \\
\hline 33 & PDSCC & $13 / 9$ & $55 / 46$ & $17 / 30$ & $15 / 15$ & $87 / 91$ \\
\hline 34 & PDSCC & $14 / 30$ & $41 / 29$ & $23 / 30$ & $22 / 11$ & $86 / 70$ \\
\hline 35 & PDSCC & $14 / 6$ & $40 / 41$ & $20 / 24$ & $26 / 31$ & $86 / 94$ \\
\hline 36 & PDSCC & $28 / 40$ & $28 / 25$ & $27 / 23$ & $17 / 12$ & $72 / 60$ \\
\hline 37 & PDSCC & $32 / 40$ & $39 / 26$ & $25 / 24$ & $4 / 10$ & $68 / 60$ \\
\hline 38 & PDSCC & $12 / 25$ & $58 / 52$ & $12 / 12$ & $18 / 11$ & $88 / 75$ \\
\hline 39 & PDSCC & $32 / 24$ & $47 / 55$ & $10 / 12$ & $11 / 9$ & $68 / 76$ \\
\hline 40 & PDSCC & $40 / 42$ & $20 / 17$ & $27 / 26$ & $13 / 15$ & $60 / 58$ \\
\hline
\end{tabular}

WDSCC, well-differentiated squamous cell carcinoma; MDSCC, moderately differentiated squamous cell carcinoma; PDSCC, poorly differentiated squamous cell carcinoma; CEP, centromere of chromosome.

significantly increased in the metastatic lymph node group, as compared with the non-metastatic group (CEP 3, $\mathrm{P}=0.0001$; CEP 17, $\mathrm{P}=0.012$ ) (Table IV).

Results of differentiation degree of CEP3 and CEP17 in three groups. The polyploidy rates for CEP3 and 17 were 35.38 and
$30.92 \%$ in well-differentiated ESCC, 55.81 and $44.43 \%$ in moderately differentiated ESCC, and 76.27 and $71.90 \%$ in poorly differentiated ESCC, respectively. Significant differences were detected between the poorly differentiated, moderately differentiated and well-differentiated ESCC specimens (CEP 3, P<0.05; CEP 17, P<0.05) (Table V). 
Table IV. CEP3 and CEP17 aberrations correlated with lymph node metastasis.

\begin{tabular}{lcc}
\hline Lymph node metastasis & CEP3 & CEP17 \\
\hline Negative & $44.38 \pm 3.820$ & $40.57 \pm 3.486$ \\
Positive & $66.32 \pm 3.946$ & $56.21 \pm 4.898$ \\
$T$-value & -3.990 & -2.639 \\
$P$-value & 0.000 & 0.012 \\
\hline
\end{tabular}

CEP, centromere of chromosome.

Table V. Differentiation degree of CEP3 and CEP17 in the three groups.

\begin{tabular}{lcc}
\hline Differentiation & CEP3 & CEP17 \\
\hline WD & $35.3846 \pm 12.2647$ & $30.9231 \pm 10.9199$ \\
MD & $55.8125 \pm 15.3849$ & $44.4375 \pm 14.5692$ \\
PD & $76.2727 \pm 9.5403$ & $71.9091 \pm 12.0785$ \\
$F$-value & 8.616 & 7.975 \\
$P$-value & $<0.05$ & $<0.05$
\end{tabular}

CEP, centromere of chromosome; WD, well-differentiated; MD, moderately differentiated; $\mathrm{PD}$, poorly differentiated.

\section{Discussion}

The incidence of EC is high in the Xinjiang Uyghur Autonomous Region of northwestern China, and the Kazakh ethnic group in particular suffers from an increased incidence of EC, as compared with other ethnic groups (2). Therefore, improved diagnosis and treatment of EC is required to enhance the survival rate and quality of life of patients with EC.

Previous studies have demonstrated that various genetic mutation occur during carcinogenesis $(10,15)$. FISH is a sensitive and specific diagnostic technique that is capable of detecting various types of cytogenetic alterations, including aneusomy, amplification and deletion. This technique has become a widely used diagnostic method in cytogenetic studies $(11,19)$; however, to the best of our knowledge there are no previous studies investigating the application of FISH in the diagnosis of Kazakh esophageal cancer.

Human chromosome 3 carries hyperdense genes, which are associated with numerous types of cancer, including raf-1 and erb-A; whereas chromosome 17 carries certain oncogenes and tumor suppressor genes, including p53, c-erbB2, BRCA1 and $n$ m23 $(9,14,20)$. Previous studies have demonstrated elevated aberration rates in CEP 3 and 17 in patients with EC, which indicates that $\mathrm{EC}$ may be correlated with constitutional cytogenetic alterations $(12,20)$.

In the present study, CEP 3 and 17 DNA probes were selected, and the cut-off value for the percentage of hyperdisomic cells was set at $10 \%$. Normal cells often have $<6 \%$ hyperdisomic cells and this discrepancy is likely to be due to sister chromatids being counted as copies $(6,8)$.
In a previous study conducted by Fiegl et al (21) FISH was applied in the diagnosis of lung, breast, liver and stomach cancer, and the confirmed diagnostic rate was increased. Furthermore, Fritcher et al (22) analyzed esophageal adenocarcinoma using the FISH method with c-Myc, P16, HER2 and $20 \mathrm{q} 13$ centromeric region probes, and demonstrated that the sensitivity of cytology was only $45 \%$ for the detection of esophageal adenocarcinoma; however, a detection rate of $100 \%$ was achieved using FISH.

In the present study, FISH was applied to Kazakh patients with ESCC to elucidate the diagnostic value of FISH in the detection of cancer cells and as a prognostic indicator. Polyploidy of CEP 3 and 17 was detected in all 40 ESCC specimens and significant differences in the rates of polyploidy were detected between the poorly differentiated, moderately differentiated and well-differentiated ESCC specimens for both CEPs (CEP 3, P<0.05; CEP 17, P<0.05). Furthermore, polyploidy was significantly increased in the metastatic lymph node group, as compared with the non-metastatic group (CEP 3, P=0.0001; CEP 17, $\mathrm{P}=0.012$ ). The average mutation rates of CEP 3 and 17 were 61.2 and 50.95\%, respectively.

The results of the present study demonstrated that the aberration rates of CEP 3 and 17 were correlated with the level of ESCC differentiation. This may due to the eating habits of the Kazakh population, in particular the over consumption of smoked meat, fermented food, heavy smoking or drinking, and the reduced consumption of fresh fruits and vegetables $(2,23,24)$.

In conclusion, the present study successfully used CEP 3 and 17 probes to detect cancerous cells in Kazakh patients with ESCC. In particular, aneuploidy was significantly higher in poorly differentiated squamous cells and the metastatic lymph node group. Therefore, DNA probes may be used as predictive biological markers for the prognosis of patients with ESCC. Furthermore, as an objective and qualitative method, FISH technology is capable of detecting CEP 3 and 17 variations in the diagnosis of Kazakh patients with ESCC, which may be used to genetically diagnose EC in the future. Further studies are required.

\section{Acknowledgements}

This study was supported by the Returned Overseas Students to Science and Technology Activities Fund (no. 2012-111) and National Natural Science Foundation of China (no. 81160279). The authors thank the Department of Hematology at the First Affiliated Hospital of Xinjiang Medical University for technical support.

\section{References}

1. Parkin DM: Global cancer statistics in the year 2000. Lancet Oncol 2: 533-543, 2001.

2. Lu XM, Zhang YM, Lin RY, Arzi G, Wang X, Zhang YL, Zhang Y, Wang Y and Wen H: Relationship between genetic polymorphisms of metabolizing enzymes CYP2E1, GSTM1 and Kazakh's Esophageal squamous cell cancer (ESCC) in Xinjiang, China. World J Gastroenterol 11: 3651-3654, 2005.

3. Krasna MJ: Multimodality therapy for esophageal cancer. Oncology (Williston Park) 24: 1134-1138, 2010.

4. Odze RD: Barrett esophagus: Histology and pathology for the clinician. Nat Rev Gastroenterol Hepatol 6: 478-490, 2009.

5. Yerian L: Histology of metaplasia and dysplasia in Barrett's esophagus. Surg Oncol Clin N Am 18: 411-422, 2009. 
6. Papanicolaou GN: Criteria of malignancy. In: Atlas of Exfoliative Cytology. Harvard University Press, Cambridge, MA: pp13-21, 1954.

7. Awut I, Niyaz M, Huizhong X, Biekemitoufu H, Yan ZH, Zhu Z, Sheyhedin I, Changmin Z, Wei Z and Hao W: Genetic diagnosis of patients with esophageal cancer using FISH. Oncol Lett 1: 809-814, 2010.

8. Niyaz M, Turghun A, Ping ZH, Zhu Z, Sheyhedin I, Ren C and Awut I: TP53 gene deletion in esophageal cancer tissues of patients and its clinical significance. Mol Med Rep 7: 122-126, 2013.

9. Rajagopalan $\mathrm{H}$ and Lengauer C: Aneuploidy and cancer. Nature 432: 338-341, 2004

10. Chao WR, Lee MY, Lin WL, Koo CL, Sheu GT and Han CP: Assessing the HER2 status in mucinous epithelial ovarian cancer on the basis of the 2013 ASCO/CAP guideline update. Am J Surg Pathol 38: 1227-1234, 2014

11. Halling KC and Kipp BR: Fluorescence in situ hybridization in diagnostic cytology. Hum Pathol 38: 1137-1144, 2007.

12. Tafe LJ, Allen SF, Steinmetz HB, Dokus BA, Cook LJ, Marotti JD and Tsongalis GJ: Automated processing of fluorescence in-situ hybridization slides for HER2 testing in breast and gastro-esophageal carcinomas. Exp Mol Pathol 97: 116-119, 2014.

13. Prins MJ, Ruurda JP, van Diest PJ, van Hillegersberg R and ten Kate FJ: Evaluation of the HER2 amplification status in oesophageal adenocarcinoma by conventional and automated FISH: A tissue microarray study. J Clin Pathol 67: 26-32, 2014.

14. Gordon MA, Gundacker HM, Benedetti J, Macdonald JS Baranda JC, Levin WJ, Blanke CD, Elatre W, Weng P, Zhou JY, et al: Assessment of HER2 gene amplification in adenocarcinomas of the stomach or gastroesophageal junction in the INT-0116/SWOG9008 clinical trial. Ann Oncol 24: $1754-1761,2013$

15. Yoshizawa A, Sumiyoshi S, Sonobe M, Kobayashi M, Uehara T, Fujimoto M, Tsuruyama T, Date H and Haga H: HER2 status in lung adenocarcinoma: A comparison of immunohistochemistry, fluorescence in situ hybridization (FISH), dual-ISH, and gene mutations. Lung Cancer 85: 373-378, 2014.
16. Awut I, Niyaz M, Biekemitoufu H, Zhang Z, Sheyhedin I and Hao W: Molecular pathological diagnosis for early esophageal cancer in Kazakh patients. Oncol Lett 3: 549-553, 2012.

17. Nakamura H, Saji H, Idiris A, Kawasaki N, Hosaka M, Ogata A, Saijo T and Kato H: Chromosomal instability detected by fluorescence in situ hybridization in surgical specimens of non-small cell lung cancer is associated with poor survival. Clin Cancer Res 9: 2294-2299, 2003.

18. Rice TW, Blackstone EH and Rusch VW: 7th edition of the AJCC Cancer Staging Manual: esophagus and esophagogastric junction. Ann Surg Oncol 17: 1721-1724, 2010.

19. Li C, Ba J, Hao X, Zhang S, Hu Y, Zhang X, Yuan W, Hu L, Cheng T, Zetterberg A, et al: Multi-gene fluorescence in situ hybridization to detect cell cycle gene copy number aberrations in young breast cancer patients. Cell Cycle 13: 1299-1305, 2014.

20. Nakamura H, Idiris A, Kawasaki N, Taguchi M, Ohira T and Kato H: Quantitative detection of lung cancer cells by fluorescence in situ hybridization: Comparison with conventional cytology. Chest 128: 906-911, 2005.

21. Fiegl M, Massoner A, Haun M, Sturm W, Kaufmann H, Hack R, Krugmann J, Fritzer-Szekeres M, Grünewald K and Gastl G: Sensitive detection of tumour cells in effusions by combining cytology and fluorescence in situ hybridisation (FISH). Br J Cancer 91: 558-563, 2004

22. Fritcher EG, Brankley SM, Kipp BR, Voss JS, Campion MB, Morrison LE, Legator MS, Lutzke LS, Wang KK, Sebo TJ and Halling KC: A comparison of conventional cytology, DNA ploidy analysis, and fluorescence in situ hybridization for the detection of dysplasia and adenocarcinoma in patients with Barrett's esophagus. Hum Pathol 39: 1128-1135, 2008.

23. Bahmanyar S and Ye W: Dietary patterns and risk of squamous-cell carcinoma and adenocarcinoma of the esophagus and adenocarcinoma of the gastric cardia: A population-based case-control study in Sweden. Nutr Cancer 54: 171-178, 2006.

24. Morita M, Kumashiro R, Kubo N, Nakashima Y, Yoshida R, Yoshinaga K, Saeki H, Emi Y, Kakeji Y, Sakaguchi Y, et al: Alcohol drinking, cigarette smoking, and the development of squamous cell carcinoma of the esophegus: Epidemiology, clinical findings, and prevention. Int J Clin Oncol 15: 126-134, 2010. 